\title{
Impact Force Measurement of a Spherical Body Dropping onto a Water Surface from Different Heights
}

\author{
Ryosuke Araki $^{\mathrm{a},},{ }^{*}$, Akihiro Takita $^{\mathrm{a}}$, Tsuneaki Ishima ${ }^{\mathrm{a}}$, Edwin Carcasona ${ }^{\mathrm{b}}$, Yusaku Fujii $^{\mathrm{a}}$ \\ ${ }^{a}$ School of Science and Technology, Gunma University, Kiryu, Tenjin-cho 1-5-1, 376-8515, Japan \\ ${ }^{\mathrm{b}}$ Faculty of Engineering, University of San Carlos, P. del Rosario Street, Cebu City 6000, Philippines \\ *Corresponding Author: ryosuke.araki.tech@gmail.com
}

\begin{abstract}
The impact force of spherical body dropping onto a water surface is measured using the method which has been proposed by us. In the method, a cube corner prism is embedded in a metal spherical body. The optical center of the cube corner prism coincides with The center of gravity of the spherical body. The motion of the spherical body is measured in the optical interferometer. The velocity of the spherical body can be calculated from the Doppler frequency measured in the interferometer. The acceleration, displacement, and inertial force of the spherical body can be calculated from the velocity of the spherical body. The dropping distance of the spherical body is variable. In this experiment, the distance is set to approximately $60 \mathrm{~mm}$, $150 \mathrm{~mm}$, and $190 \mathrm{~mm}$, so that the difference of impact force caused by the difference of initial velocity is evaluated in detail.
\end{abstract}

Keywords: water entry, the Levitation Mass Method, embedded cube-corner prism.

\section{Introduction}

Water impact, water entry, or slamming is a complex nonlinear phenomenon, which is especially important in the fields of naval architecture, ocean engineering, aerospace engineering, and fluid engineering. Although the first analytical solution with the added mass concept was proposed in $1929^{(1)}$, this nonlinear problem is still attracting great attention ${ }^{(2)}$. There have been many studies on this problem; some employed numerical simulation ${ }^{(3-6)}$, others analyzed images taken by high-speed cameras were analyzed $^{(7)}$. However, it has been difficult to measure the mechanical quantities precisely, such as the impact force acting upon the object from the water surface.

We have previously developed a method for precise mechanical measurement known as the Levitation Mass Method (LMM). In the LMM, a mass levitated by an aerostatic linear bearing is used, and the laser Doppler interferometer is used for measuring the velocity. We have precisely calibrated force transducers ${ }^{(8)}$, tested materials ${ }^{(9,10)}$, and examined the structures ${ }^{(11)}$ using LMM. A method to measure the impact force of a spherical body dropping onto the water surface has been proposed by modifying the $\mathrm{LMM}^{(12)}$. In the method, a cube corner prism is inserted in the stainless spherical body and the inertial force of the spherical body is measured using the laser Doppler interferometer. The acceleration, displacement, and inertial force of the spherical body are measured precisely in the study.

In this paper, the dropping distance of the spherical body is changed, so that the velocity of water entry of the spherical body is changed, and the phenomenon of the collision between the spherical body and water surface is evaluated in detail.

\section{Experiment}

Figure 1 shows a schematic diagram of the experimental setup for evaluating the impact force acting on a spherical body that drops onto a water surface. The main part of the body is fabricated by machining a spherical SUS440 stainless steel body approximately $30.2 \mathrm{~mm}$ in diameter, the surface of which tempered. A cube corner prism, $12.7 \mathrm{~mm}$ in diameter, is inserted into the machined hole of the spherical body with an adhesive agent in order to make its optical center coincide with the center of gravity of the entire body. The total mass of the entire body, $\boldsymbol{M}$, is 
approximately $93.88 \mathrm{~g}$. The origins of the time $(\boldsymbol{t}=0)$ and position $(\boldsymbol{x}=0)$ axes are set to be the time and the position at which the impact force is first detected in the water test. The direction of the coordinate system for the velocity, the acceleration, and the force acting on the body is upward in Fig. 1, which is opposite direction of gravity.

The total force acting on the spherical body can be considered as the product of its mass and acceleration; i.e., $\boldsymbol{F}_{\text {mass }}=\boldsymbol{M a}$. Differentiating the body's velocity which is calculated from the measured value of the Doppler shift frequency of the signal beam of the interferometer, the acceleration is calculated. The signal beam of interferometer $f_{\text {Doppler }}$ and the velocity of the spherical body $v$ is expressed as:

$$
\begin{aligned}
& f_{\text {Doppler }}=-\left(f_{\text {beat }}-f_{\text {rest }}\right) \\
& v=\lambda_{\text {air }}\left(f_{\text {Doppler }}\right) / 2
\end{aligned}
$$

where $\lambda_{\text {air }}$ is the wavelength of the signal beam, $f_{\text {beat }}$ is the beat frequency, and $f_{\text {rest }}$ is the rest frequency. The beat frequency $f_{\text {beat }}$ is the difference of the frequency between the signal beam and the reference beam. The rest frequency $f_{\text {rest }}$ is equivalent to the beat frequency $f_{\text {beat }}$ when the spherical body is at rest and the signal beam is not Doppler
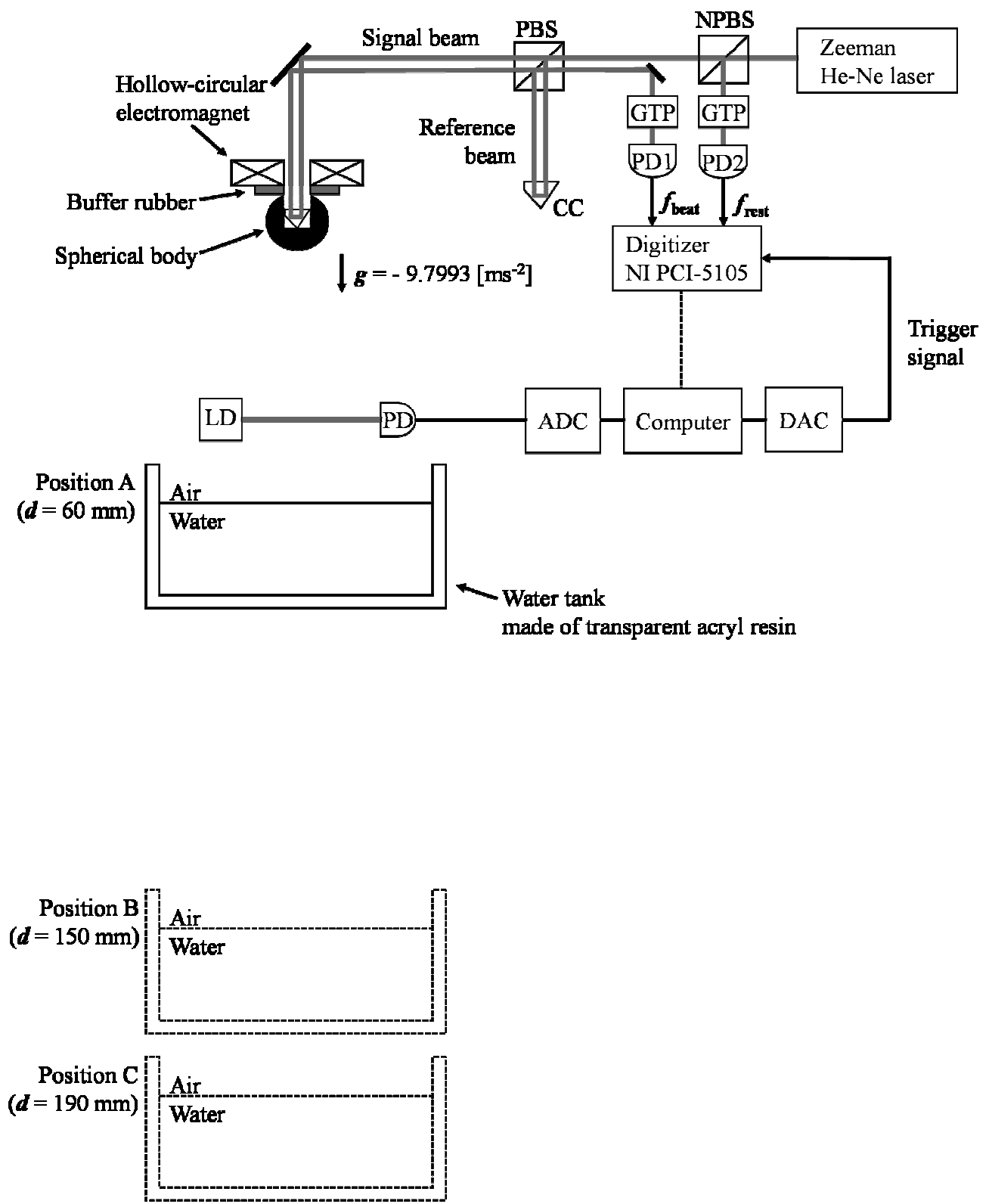

Fig. 1. Experimental setup. Code: $\mathrm{CC}=$ cube-corner prism, $\mathrm{PBS}=$ polarizing beam splitter, NPBS = non-polarizing beam splitter, GTP = Glan-Thompson prism, $\mathrm{PD}=$ photodiode, $\mathrm{LD}=$ laser diode, $\mathrm{ADC}=$ analogue-to-digital converter, DAC = digital-to-analogue converter. 
shifted.

The total force, $\boldsymbol{F}_{\text {mass }}$, consists of the gravitational force acting upon the body, $-\boldsymbol{M g}$, and the impact force acting from the water, $\boldsymbol{F}_{\text {water, }}$, if other forces, such as the air drag and the magnetic force from the hollow-circular electromagnet, are negligible. If the experiment is in such a situation, the total force can be expressed as:

$$
F_{\text {mass }}=-M g+F_{\text {water }}
$$

where $\boldsymbol{g}$ is the acceleration of gravity, approximately 9.799 $\mathrm{m} / \mathrm{s}^{2}$ in the Kiryu, Gunma, Japan. Therefore, the impact force acting from the water can be calculated as:

$$
F_{\text {water }}=F_{\text {mass }}+M g
$$

An optical interferometer is used to accurately measure the velocity as shown in Fig. 1. A Zeeman-type two-wavelength He-Ne laser in is used as the light source. Each beam has different frequency and orthogonal polarization. The difference between the two frequencies; i.e., the rest frequency $f_{\text {rest }}$, is approximately $3.0 \mathrm{MHz}$.
A digitizer (NI PCI-5105, National Instruments Corp., USA) records the output signals of PD1 and PD2 with a sample number of $5 \mathrm{M}$ for each channel, a sampling rate of $30 \mathrm{M}$ samples per second, and a resolution of 8 bit. Therefore the measurement duration of the digitizer is approximately $0.167 \mathrm{~s}$ in this experiment. The frequencies $f_{\text {beat }}$ and $f_{\text {rest }}$ are accurately determined from the digitized waveforms of the output signals appearing at PD1 and PD2, respectively, using the recently developed Zero-Crossing Fitting method (ZFM). In ZFM, all zero-crossing times inside each sampling interval are used to determine the frequency $^{(13)}$. In our analysis, the sampling interval is defined by $\mathrm{N}=1000$ periods of the signal waveform, which corresponds to $0.125 \mathrm{~ms}$ when $f_{\text {beat }}$ is approximately $8 \mathrm{MHz}$.

In the experiment, the spherical body is held and released using a hollow-circular electromagnet. The electromagnet is turned on/off manually. Three sets of measurements are conducted by the difference of the initial position from the water surface. 5 drop measurements are
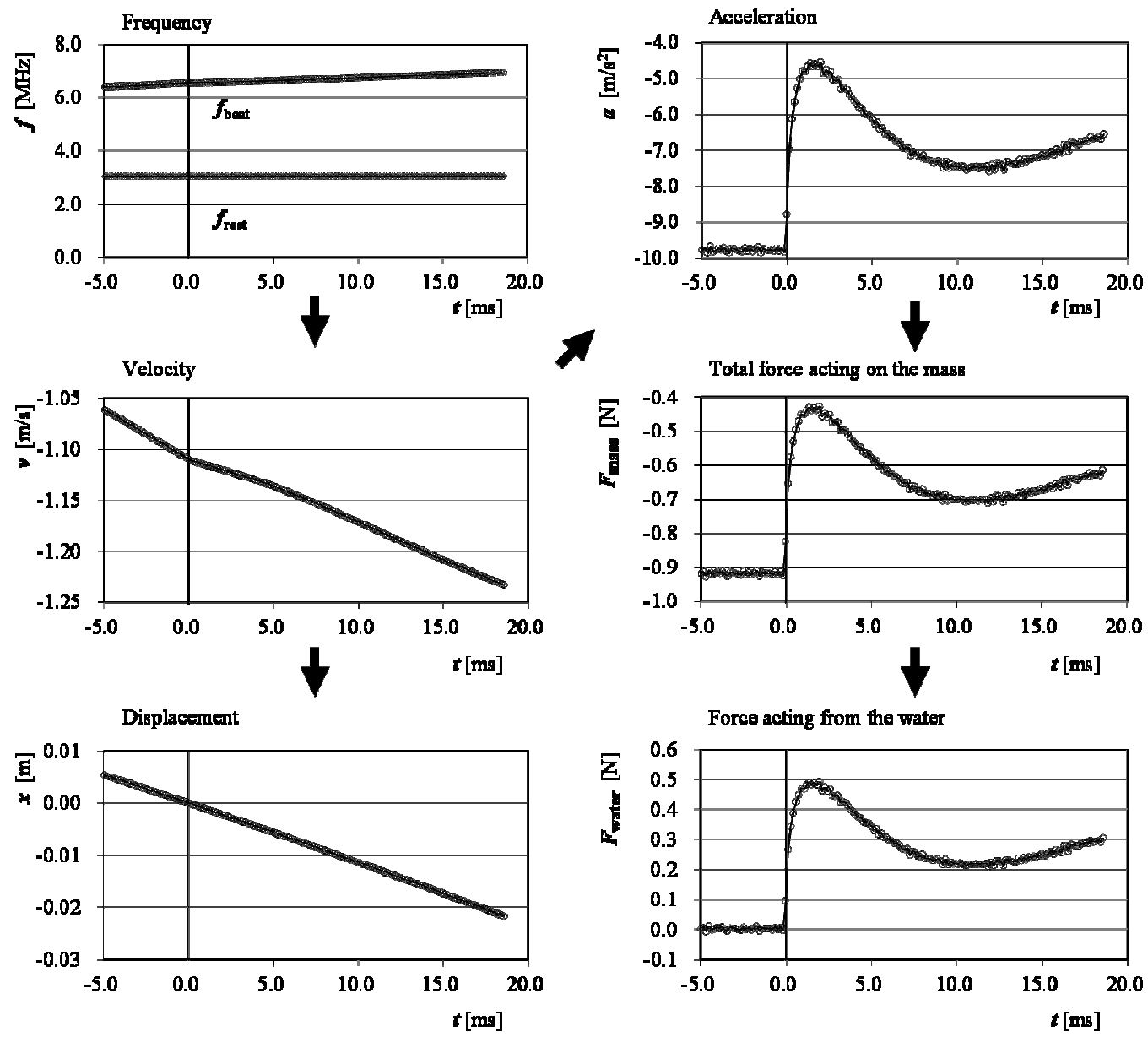

Fig. 2. Data processing procedure: Calculation of velocity, position, acceleration, and force from frequency. 


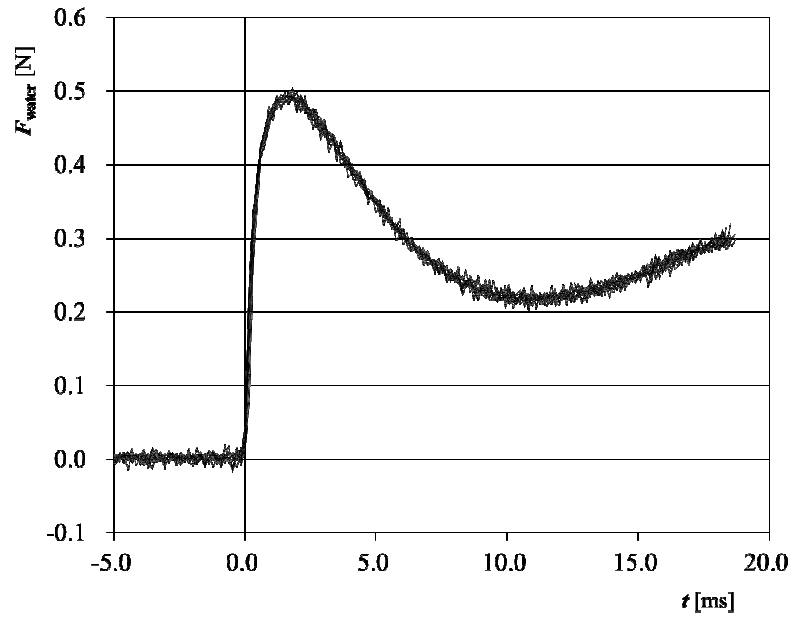

(a) Position A.

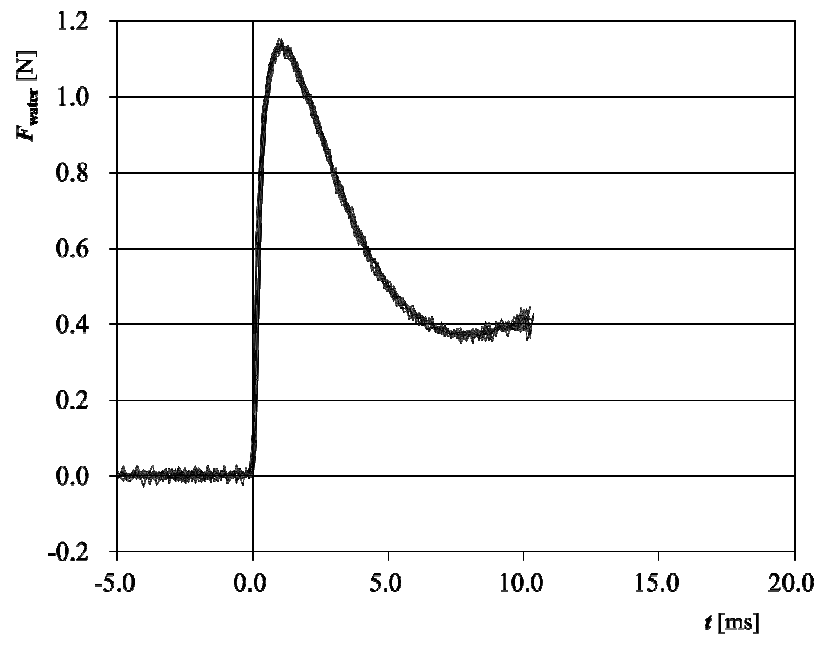

(b) Position B.

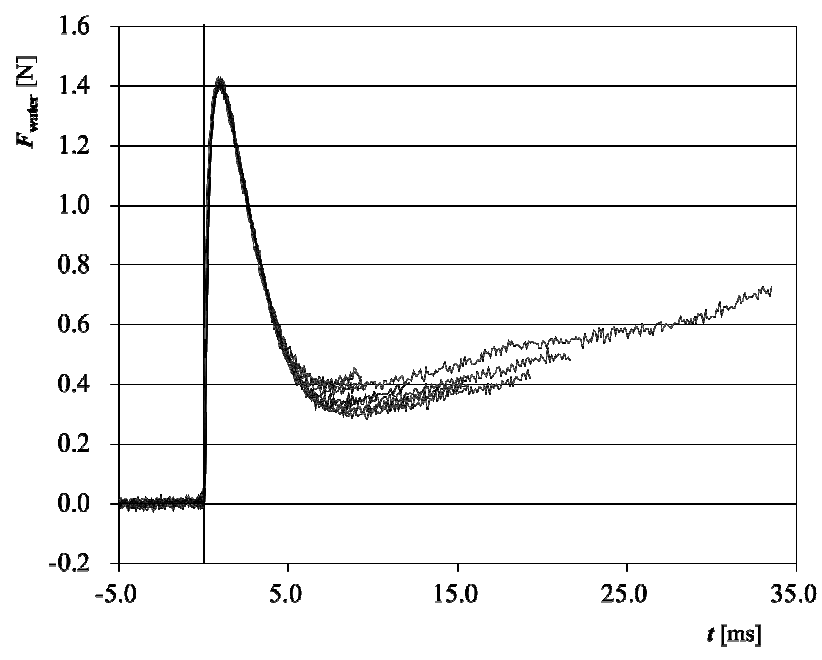

(c) Position C.

Fig. 3. Changes in impact force against time of each set of measurement. taken in each set of measurements. The dropping distances $\boldsymbol{d}$ are approximately 60, 150, and $190 \mathrm{~mm}$, which are named position $\mathrm{A}, \mathrm{B}$, and $\mathrm{C}$ in this paper, respectively. The height $60 \mathrm{~mm}$ is the shortest distance that we are able to set, and the velocity of the spherical body is not able to be measured using the digitizer if the distance is longer than $190 \mathrm{~mm}$ due to Nyquist frequency of the digitizer. The digitizer is initiated by a sharp trigger signal generated using a digital-to-analog converter (DAC: NI PCI-6221, National Instruments Corp., USA). This signal is activated using a light switch, which is a combination of a laser diode and a photodiode.

\section{Results}

Figure 2 shows the data processing procedure, in which the velocity, $\boldsymbol{v}$; the position, $\boldsymbol{x}$; the acceleration, $\boldsymbol{a}$; the total force acting upon the body, $\boldsymbol{F}_{\text {mass }}$; and the impact force acting upon the body from the water, $\boldsymbol{F}_{\text {water }}$ are calculated from the obtained frequencies $f_{\text {beat }}$ and $f_{\text {rest }}$.

The figure shows data from the point, in which the light switch is activated, to the point, in which the beam strength becomes below $75 \%$ of the maximum beam strength. The measurement is cut off in which the beam is interrupted by the water splash.

Figure 3 shows the changes in $\boldsymbol{F}_{\text {water }}$ against time for the 5 drop measurements in each measurement set. For each of the measurement sets, the results coincide very well. The results indicate a high reproducibility of the measurements. Although the cutting off time of the results of the position $\mathrm{C}$ varies widely from approximately $9.32 \mathrm{~ms}$ to $33.53 \mathrm{~ms}$, the changes in $\boldsymbol{F}_{\text {water }}$ near the peak coincide well.

Figure 4 shows the changes in $\boldsymbol{F}_{\text {water }}$ against the time in each measurement set. Figure 5 shows the impact force, $\boldsymbol{F}_{\text {water }}$ against position of the spherical body $\boldsymbol{x}$. The drop experiment shown in Figure 4 is the same as that of Figure 5.

\section{Discussions}

The sources of uncertainty in determining the instantaneous value of Fwater are considered as follows:

[U.1] Optical alignment

The major source of uncertainty in the optical alignment was the inclination of the $1 \mathrm{mrad}$ signal beam; this resulted in a relative uncertainty in the inertial force of approximately $5 \times 10^{-7}$. Therefore the uncertainty in the optical alignment is negligible. 


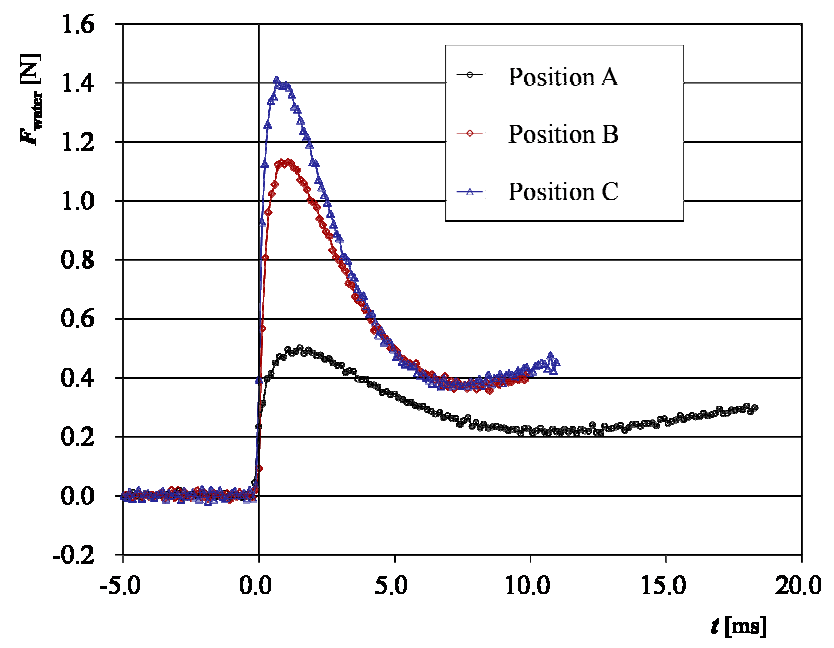

Fig. 4. Comparison of dynamic force $\boldsymbol{F}_{\text {water }}$ against time $\boldsymbol{t}$ between the position A, B, and C.

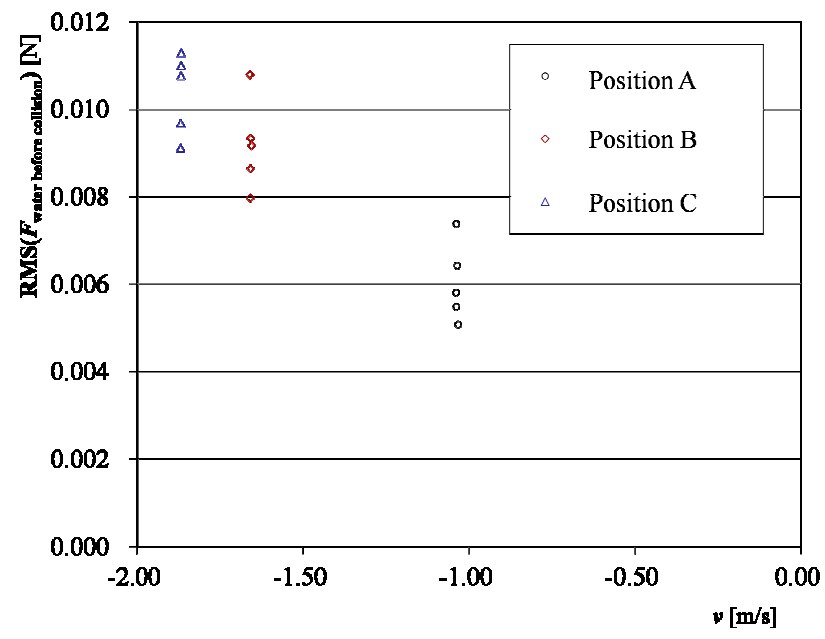

Fig. 6. The relationship between the RMS value of $\boldsymbol{F}_{\text {water }}$ in the free-fall motion and the velocity.

\section{[U.2] Mass calibration}

The uncertainty in the mass measurement when using the electric balance was approximately $0.01 \mathrm{~g}$, which is $0.01 \%$ of the total mass of the moving part. This corresponds to $0.1 \mathrm{mN}$ when the applied force reaches its maximum value of position $C \boldsymbol{F}_{\text {mass, }}$ max, position $\mathbf{C}=1.4 \mathrm{~N}$, which is negligible.

\section{[U.3] Acceleration due to gravity}

The acceleration due to gravity $\boldsymbol{g}$ is estimated to be $9.799 \mathrm{~m} / \mathrm{s}^{2}$ in the room which is used for this experiment. The value of gravity acceleration includes an uncertainty of $0.01 \%$, which is negligible.

[U.4] Noise of the optical interferometer

[U.5] Frequency estimation using the ZFM

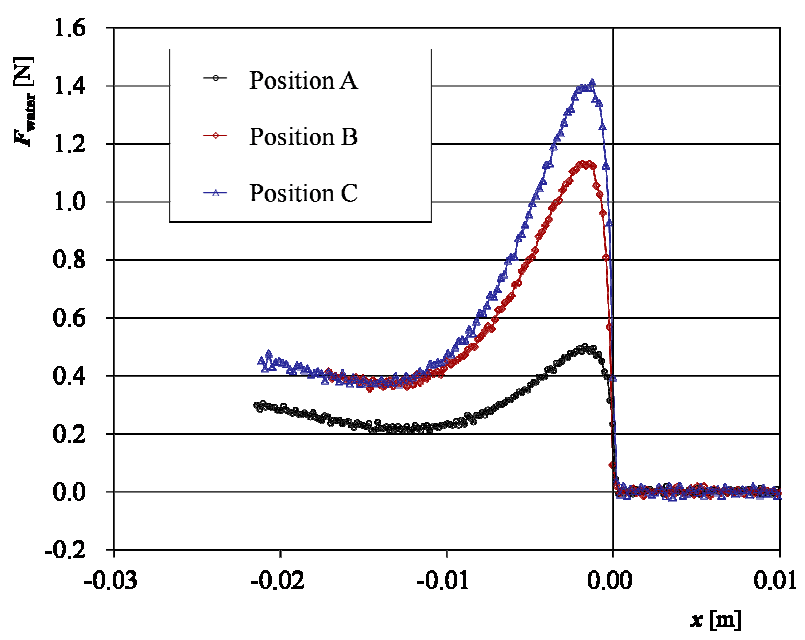

Fig. 5. Comparison of dynamic force $\boldsymbol{F}_{\text {water }}$ against the displacement $\boldsymbol{x}$ between position A, B, and C.

[U.6] Numerical calculation of force from the frequency

The sources of uncertainty [U.4], [U.5], and [U.6] are difficult to estimate separately. However, the uncertainty from each component is assumed to be similar between when in free-fall motion before the collision and during the collision with water. The mean and standard deviation of $\boldsymbol{F}_{\text {water }}\left(=\boldsymbol{F}_{\text {mass }}+\boldsymbol{M g}\right)$ during free-fall motion before the collision, at height above the water surface $10-50 \mathrm{~mm}$, is not more than $1.6 \mathrm{mN}$ and $13 \mathrm{mN}$, respectively, for the 7 drop measurements. Therefore, the combined uncertainty of [U.4]-[U.6] is estimated to be approximately this standard deviation, $13 \mathrm{mN}$.

Since only [U.4]-[U.6] have a significant contribution, the total uncertainty when determining the instantaneous value of the force $\boldsymbol{F}_{\text {mass }}$ acting on the spherical body under test is estimated to be $13 \mathrm{mN}$. This corresponds to $1 \%$ of the maximum force of position $\mathrm{C}$ of approximately $1.4 \mathrm{~N}$.

Figure 6 shows the relationship between the RMS value of $\boldsymbol{F}_{\text {water }}$ in the free-fall motion and the velocity when the spherical body collides with the water surface which shows the effect of air drag. The force acting on the spherical body during free-fall motion is related to the velocity due to air turbulence. The effect of air turbulence is considered more carefully in the future.

In the experiment, the relationship between the instantaneous values of the impact force, the position, and the velocity of the spherical body dropping onto a water surface are accurately evaluated. These accurate measured data are valuable as reference data in evaluating the validity and efficiency of numerical simulation methods. 


\section{Conclusions}

The impact force of a spherical body dropping on a water surface is evaluated in detail. The 3 dropping distances are set. The velocity of the center of gravity of a metal spherical body is accurately measured using an optical interferometer so that the impact force acting on the spherical body from a water surface is accurately calculated in each distance. The displacement is also calculated from the frequency obtained in the interferometer.

\section{Acknowledgment}

This work was supported in part by a research-aid fund of the Asahi Glass Foundation, a research-aid fund of the NSK Foundation for the Advancement of Mechatronics (NSK-FAM) and the Grant-in-Aid for Scientific Research (B) 24360156 (KAKENHI 24360156).

\section{References}

(1) T. Kármán : "The Impact on Seaplane Floats during Landing", National Advisory Committee for Aeronautics Technical Notes, No. 321, 1929

(2) C.M. Seddon, and M. Moatamedi : "Review of water entry with applications to aerospace structures", International Journal of Impact Engineering, Vol. 32, pp. 1045-1067, 2006

(3) H. Sun, and O.M. Faltinsen : "Water impact of horizontal circular cylinders and cylindrical shells", Applied Ocean Research, Vol. 28, pp. 299-311, 2006

(4) Q. Yang, and W. Qiu : "Numerical simulation of water impact for 2D and 3D bodies", Ocean Engineering, Vol. 43, pp. 82-89, 2012

(5) H.B. Gu, L. Qian, D.M. Causon, C.G. Mingham, and P. Lin : "Numerical simulation of water impact of solid bodies with vertical and oblique entries", Ocean Engineering, Vol. 75, pp. 128-137, 2014

(6) M. Ahmadzadeha, B. Saranjam, A.H. Fard, and A.R. Binesh : "Numerical simulation of sphere water entry problem using Eulerian-Lagrangian method", Applied Mathematical Modelling, Vol. 38, pp. 1673-1684, 2014

(7) H. Tabuteau, D. Sikorski, S.J. Vet, and J.R. Bruyn : "Impact of spherical projectiles into a viscoplastic fluid", Physical Review, Vol. 84, pp. 031403 1-10, 2010

(8) Y. Fujii : "Toward Establishing Dynamic Calibration Method for Force Transducers", IEEE Transactions on
Instrumentation and Measurement, Vol. 58, pp. 2358-2364, 2009

(9) Y. Fujii : "Microforce materials tester", Review of Scientific Instruments, Vol. 76, pp. 065111 1-7, 2005

(10) R. Araki, K. Irisa, K. Watanabe, N. Miyashita, A. Takita, and Y. Fujii : "The Dynamic Characteristics of Gelled Food Materials against Impact Force", Vol. 2, No. 1, pp.19-23, 2014

(11) Y. Fuji, and D.W. Shu : "Impact force measurement of an actuator arm of a hard disk drive", International Journal of Impact Engineering, Vol. 35, pp. 98-108, 2008

(12) R. Araki, A. Takita, H. Kawashima, N. Pornsuwancharoen, S. Punthawanunt, E. Carcasona, and Y. Fujii : "Impact force measurement of a spherical body dropping onto a water surface", Review of Scientific Instruments, Vol. 85, pp. 075108 1-5, 2014

(13) Y. Fujii, and J.P. Hessling : "Frequency Estimation Method from Digitized Waveform", Experimental Techniques, Vol. 33, pp. 64-69, 2009. 